\title{
Der Uetliberg bleibt top
}

\section{Bruno Kesseli}

Dr. med. et lic. phil., Chefredaktor

\author{
Auch in der 10. Auflage boten die traditionellen Gesundheitstage der Ärztegesell- \\ schaft des Kantons Zürich (AGZ) auf dem Uetliberg eine stimulierende Mischung \\ von Referaten und Diskussionen. Das Spektrum reichte von Gesundheitspolitik \\ über Wissenschaft, Forschung, Technik und aktuelle medizinische Brennpunkte \\ bis hin zu ethischen und philosophischen Themen.
}

«Fortschritt und Wandel der Werte» - unter diesem Titel stand die Jubiläumsausgabe der diesjährigen Zürcher Gesundheitstage. Bereits zum 10. Mal fand das Symposium auf dem Zürcher Hausberg statt, und noch sind keine Abnützungserscheinungen ersichtlich. Im Gegenteil: Auch in diesem Jahr wurde dem Publikum ein Programm geboten, das ein breites Feld von aktuellen Themen im Zusammenhang mit dem ärztlichen Berufsfeld abdeckte, über dessen Rand aber immer wieder hinausgeschaut wurde.

\section{Leistungsfähiges Gesundheitssystem}

Anknüpfend an die von seinem Vorgänger Urs Stoffel etablierte Tradition, eröffnete AGZ-Präsident Josef Widler den Anlass mit einer gesundheitspolitischen Tour d'Horizon. Der erfahrene (Standes-)Politiker bedient die Klaviatur der politischen Rhetorik routiniert und versteht es, die Interessen der Ärzteschaft mit schla-

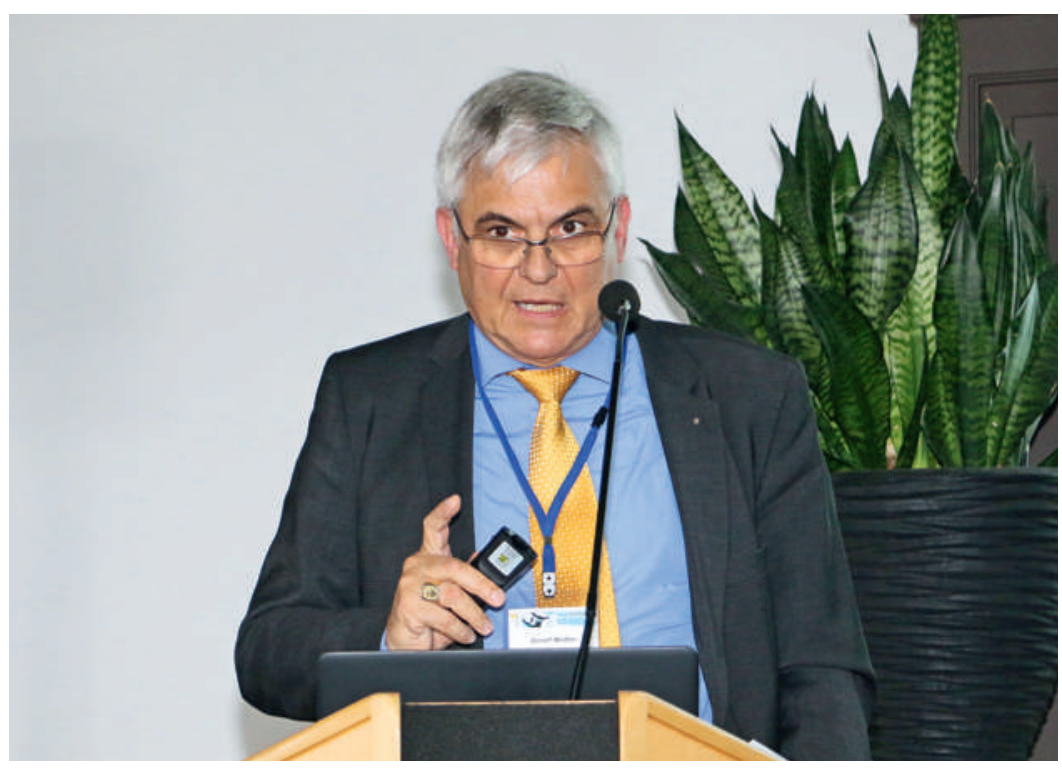

Kämpferisch: AGZ-Präsident Josef Widler bei seiner gesundheitspolitischen Tour d'Horizon. genden Argumenten zu vertreten. Anhand konkreter Beispiele zeigte er, mit wie vielen Ansprüchen sich Ärztinnen und Ärzte heutzutage konfrontiert sehen, während ihnen gleichzeitig die stetig steigenden Gesundheitskosten zumindest teilweise angelastet werden. Anhand verschiedener Grafiken machte er deutlich, dass unser Gesundheitssystem, das immer mehr alte Menschen zu versorgen hat, Beträchtliches leistet. "Ohne Ärzte kein medizinischer Fortschritt», so seine Quintessenz. Die AGZ ist gewillt, ihren Beitrag zu einer auch zukünftig guten medizinischen Versorgung der Bevölkerung leisten, beispielsweise durch die Gründung der AGZ Support AG. Diese Gesellschaft hat die Aufgabe übernommen, den ärztlichen Notfalldienst im Kanton Zürich durch eine geeignete Organisation langfristig, nachhaltig und unter Beibehaltung der heutigen Qualität sicherzustellen.

Natürlich musste auch die Revision des ambulanten Arzttarifs angesprochen werden, die für die Ärzteschaft eine beträchtliche Belastungsprobe darstellt. Als Präsident einer grossen kantonalen Ärztegesellschaft hat Josef Widler die Interessen aller Fachgruppen zu berücksichtigen. "Nur Verlierer» sah er im Entwurf, der zum Zeitpunkt des Symposiums zur Diskussion stand und inzwischen im Rahmen einer Urabstimmung von der Mehrheit der teilnehmenden FMHMitglieder abgelehnt wurde. Ein spezieller Dorn im Auge ist ihm die Kostenneutralität, die er als gesetzeswidrig bezeichnete und deren Aufhebung er forderte. Ob die AGZ mit dem von ihr angestrebten Taxpunktwert 2017 von einem Franken durchkommt, wird sich zeigen. Die Wahrscheinlichkeit, dass die Tarife auch an den nächsten Zürcher Gesundheitstagen ein Thema sein werden, ist jedenfalls hoch.

\section{Der Mensch als «komplexes Wunder»}

Wenn Ärztinnen und Ärzte durch intelligente Maschinen ersetzt würden, wären Tarifdiskussionen vermut- 


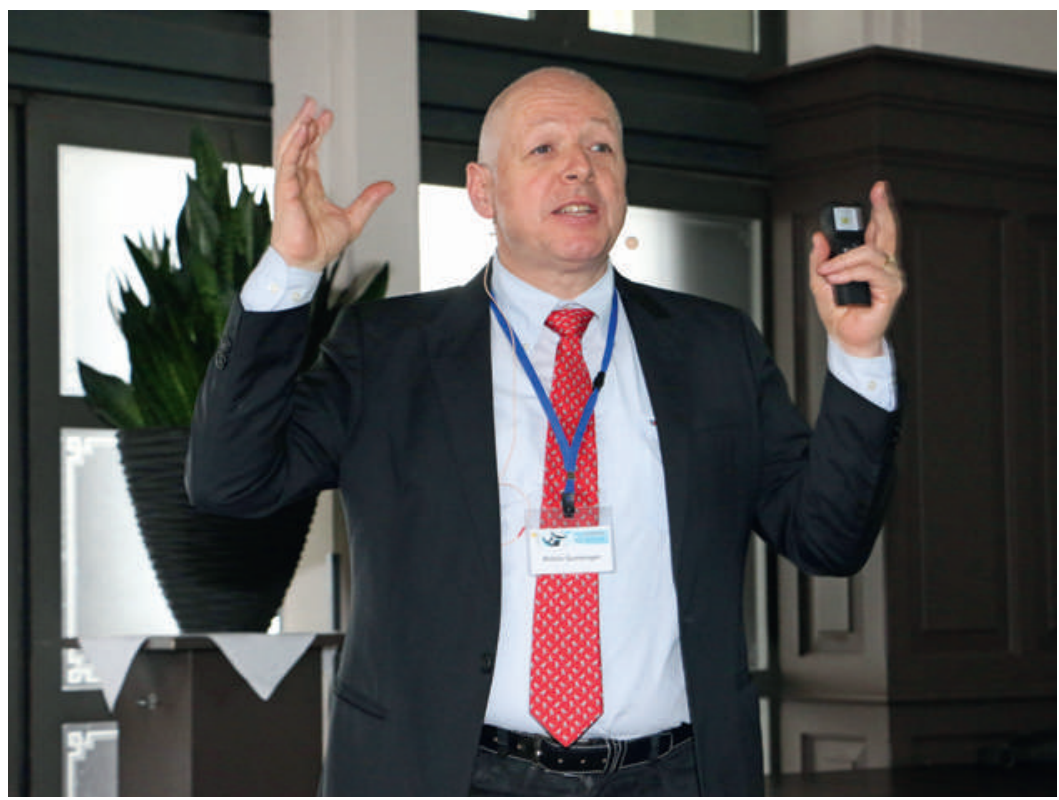

Anton Gunzinger referierte zum Thema «Mensch und Maschine».

lich kein Thema. Technikvisionäre wie der US-Amerikaner Ray Kurzweil träumen offenbar von solchen Szenarien. Anton Gunzinger, der auf dem Uetliberg zum Thema «Mensch und Maschine» sprach, wirkt diesbezüglich etwas geerdeter. Der Professor und Dozent am Departement für Informationstechnologie und Elektrotechnik der ETH Zürich und Inhaber der Firma

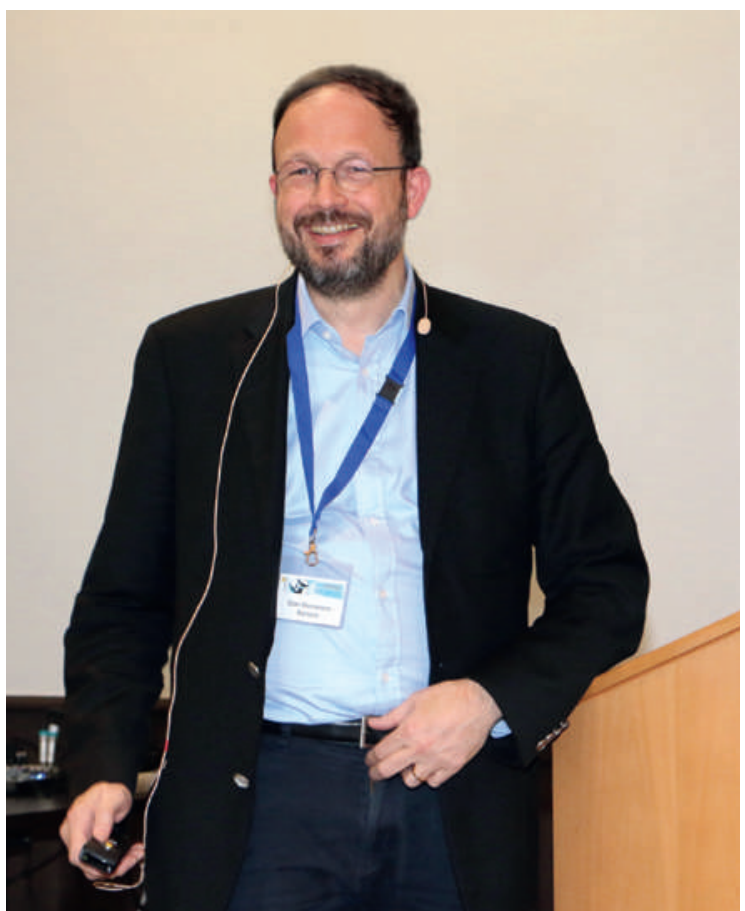

Den Patienten zuhören: Gian Domenico Borasio, Professor für Palliativmedizin in Lausanne.
Supercomputing Systems AG wurde bekannt als Entwickler eines superschnellen Rechners. Dass er die schwindelerregenden Fortschritte der Computertechnologie, speziell im Bereich selbstlernender Systeme, hautnah miterlebt und sein Geld als «Techniker» verdient, tut seinem Staunen über den Menschen, den er als «komplexes Wunder» bezeichnet, keinen Abbruch: «Da hat jemand einen ziemlich guten Job gemacht», urteilt er mit einem Anflug britischen Understatements. Das zentrale Unterscheidungsmerkmal zwischen Mensch und Maschine ortet er im «Ich-Bewusstsein». Angesichts der Komplexität lebendiger Systeme wundert sich Gunzinger darüber, wie «locker» auch in der Wissenschaft mitunter mit Risiken umgegangen werde, beispielsweise im Bereich der Gentechnologie. Die Übertragung der Industrielogik auf die Organisation von Spitälern ist für ihn aufgrund der Komplexität dieser Gebilde der falsche Weg: «Das kann nicht gutgehen.» Medizinische Organisationen sollten als lernende Systeme konzipiert werden, in denen Verantwortung soweit wie möglich nach unten delegiert werden müsste. Grosse Chancen für unser Gesundheitswesen sieht Gunzinger etwa in der Prophylaxe («Work-Life-Balance»), der Kooperation von Mensch und Maschine in Bereichen, wo dies sinnvoll ist, in der Unterstützung der Selbstheilungsprozesse und nicht zuletzt in der Integration des Todes.

\section{«Selbst bestimmt sterben»}

Um die Integration statt Verdrängung des Todes geht es auch in der Palliativmedizin. Ein äusserst engagierter Vertreter dieser Disziplin ist Gian Domenico Borasio, der an der Universität Lausanne einen Lehrstuhl für Palliativmedizin innehat. "Selbst bestimmt sterben" lautete der eigenwillig in drei Wörtern formulierte Titel seines Referats. Allein schon die demographische Entwicklung mit einer Zunahme der Demenzerkrankungen und der Sterbefälle legt nahe, dass die Palliativmedizin inskünftig an Bedeutung gewinnen wird. Diese Disziplin versucht, durch Vorbeugung und Linderung von Leiden die Lebensqualität von Patienten - und ihren Angehörigen! - zu verbessern, die mit einer lebensbedrohlichen Erkrankung konfrontiert sind. Es dürfte wohl kaum jemanden geben, der diese Zielsetzung nicht unterschreiben kann. Gemäss den Daten, die Borasio präsentierte, wird das Ziel einer höheren Lebensqualität durch palliativmedizinische Betreuung tatsächlich erreicht, und dies bei geringeren Kosten und einer signifikant höheren Lebenserwartung. Als eine Schlüsselfähigkeit von Fachpersonen, die palliativmedizinisch tätig sind, bezeichnet Borasio die Fähigkeit, den Patienten zuzuhören: 
«Die Medizin der Zukunft wird eine hörende sein, oder sie wird nicht mehr sein.»

\section{Von Ernährung über Satire bis zu «Big Data»}

Wie breit das am Symposium abgedeckte Spektrum war, lässt sich leicht anhand der Themen zeigen, die von den weiteren Referenten abgedeckt wurden. Der Soziologe Jens Krüger sprach über die Ernährung der Zukunft und stellte fest, dass Essen zunehmend zum Ausdruck des persönlichen Lebensstils und der Weltanschauung werde. «Bio und Nachhaltigkeit», aber auch «In-vitroFleisch \& Co.» würden in diesem Zusammenhang an Bedeutung gewinnen, prognostizierte er. Die Technik spiele im Zusammenhang mit Ernährungstrends eine wichtige Rolle, und wie in anderen Lebensbereichen werde die Personalisierung auch im Bereich der Lebensmittel fortschreiten - Stichwort: Personal Food.

Nachdem der Kabarettist Lorenz Kaiser den ersten Veranstaltungstag satirisch hatte ausklingen lassen, bot auch die Fortsetzung viel Überraschendes und Informatives, oftmals kombiniert. Ernst Hafen, Direktor des Instituts für Molekulare Systembiologie der ETH Zürich, baute in seine Ausführungen zu «Big Data» den Vorschlag ein, künftig analog zu Bankkonten persönliche Datenkonten einzurichten, deren Zweitverwertung den Besitzern einen finanziellen Nutzen, also eine Art Zinsen bringen könnte. Datenbesitz und Verwertungshoheit sollten per Verfassungsgesetz beim betreffenden Kontoinhaber liegen. Hafen plant dazu eine Volksinitiative und sucht Mitstreiter.

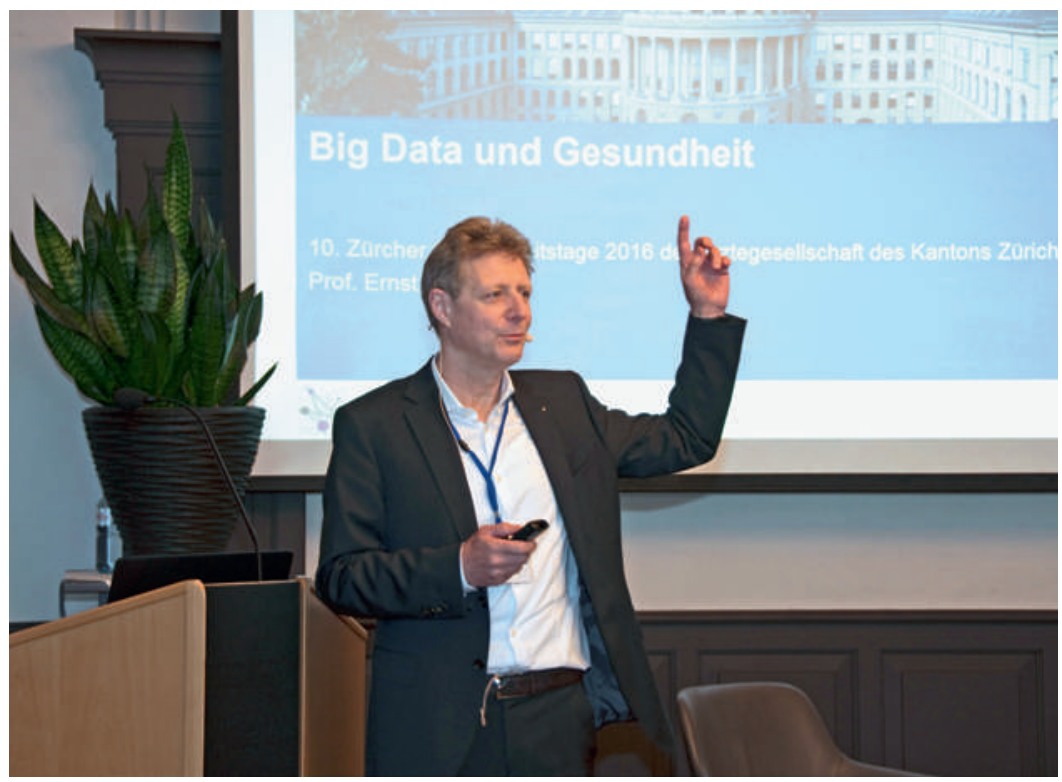

«Big Data» mit Zinsen: Ernst Hafen.

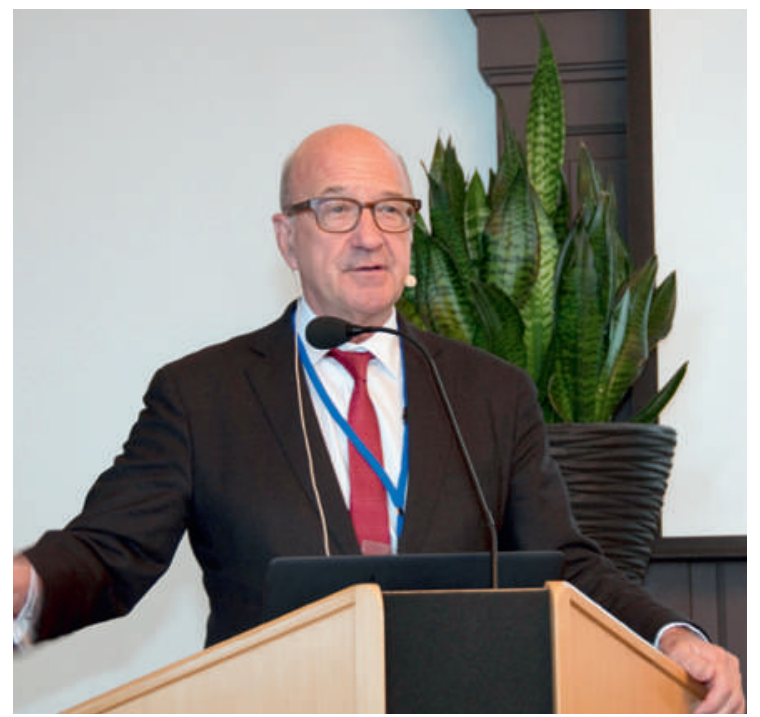

Bahnbrechende Fortschritte: Florian Holsboer sprach über personalisierte Depressionstherapie.

\section{Personalisierte Therapie psychiatrischer Erkrankungen}

Mit einem Zitat im Geist von Sokrates stieg Florian Holsboer, ehemaliger Direktor des Max-Planck-Instituts für Psychiatrie, in sein Referat ein: «If the brain were so simple that we could understand it, we would be so simple that we couldn't», hatte Edward Pugh 1973 in seinem Buch The Biological Origin of Human Values konsta-tiert. Bescheidenheit ist angesichts der nicht wirklich fassbaren Komplexität unseres Gehirns zweifellos angesagt. Dass wir dennoch daran sind, die Tür zu bahnbrechenden Fortschritten in der Behandlung psychiatrischer Erkrankungen aufzustossen, zeigte Holsboer im Folgenden am Beispiel der personalisierten Depressionstherapie. Gegen die sich abzeichnenden, auf das Individuum massgeschneiderten Behandlungen wirken die heutigen medikamentösen Therapieversuche wie ein Tappen im Dunkeln.

Die politischen und philosophischen Schlussbetrachtungen des Zürcher Gesundheitsdirektors Thomas Heiniger und des Philosophen und Publizisten Ludwig Hasler schlossen einen Anlass ab, der dem Uetliberg-Claim "Top of Zurich» in verschiedener Hinsicht gerecht wurde. Man sieht den nächsten Gesundheitstagen mit Vorfreude und einiger Erwartung entgegen.

Der Verfasser dankt Bianka Hubert, stellvertretende Generalsekretärin der AGZ, für die zusammenfassenden Notizen zum zweiten Veranstaltungstag, an dem er nicht teilnehmen konnte. 